\title{
Urgences
}

\section{Des X... à la manière toscane}

\section{Roger Magini}

Numéro 27, mars 1990

Images imaginaires

URI : https://id.erudit.org/iderudit/025573ar

DOI : https://doi.org/10.7202/025573ar

Aller au sommaire du numéro

Éditeur(s)

Urgences

ISSN

0226-9554 (imprimé)

1927-3924 (numérique)

Découvrir la revue

Citer ce document

Magini, R. (1990). Des X... à la manière toscane. Urgences, (27), 44-45.

https://doi.org/10.7202/025573ar d'utilisation que vous pouvez consulter en ligne.

https://apropos.erudit.org/fr/usagers/politique-dutilisation/ 


\section{Des $\mathrm{X}$... à la manière toscane Roger Magini}

Quand on veut bâtir un X... à la manière toscane, la longueur de la place qui lui est destinée étant divisée en six parties, il est nécessaire d'en réserver cinq pour la largeur. Après avoir partagé la longueur en deux parties, celle de derrière figurera les chapelles, et celle de devant organisera les colonnes. Il faut répartir la largeur en dix parties, dont trois à droite et à gauche, qui logeront les chapelles ou les ailes, s'il y a lieu; les quatre autres seront pour le milieu. L'espace qui préfigure le porche au devant de X... sera partagé de telle manière, afin de placer les colonnes, que celles-ci soient à angle droit des pilastres à l'extrémité des murs, et que devant ceux-ci érigés entre les pilastres et le milieu de X..., il y ait deux autres colonnes, disposées entre les pilastres [...].

La grosseur des colonnes, à la base, représente le septième de leur hauteur, et celle-ci le tiers de la largeur de $\mathrm{X}$... La colonne se rétrécit, vers le haut, du quart de la grosseur de sa base. La plinthe de la base, qui est ronde, doit être de la moitié de l'épaisseur de celle-ci, et sa moulure de la même proportion que la plinthe. La hauteur du chapiteau étant divisée en trois parties, il faut en attribuer une à la plinthe qui fait office de tailloir, la seconde à l'échine et la dernière à la gorge avec l'astragale et la moulure [...].

Il y a des X... ronds. Ceux qui possèdent des colonnes sans murailles à l'intérieur sont dits monoptères; les autres sont des périptères. Ceux qui n'ont pas de murailles sont comme un tribunal vers lequel on monte; ils ont le tiers du diamètre de $\mathrm{X}$...

Si X... est périptère, les piédestaux sont posés sur deux degrés, et la muraille distante de ceux-ci d'environ le cinquième de $\mathrm{X}$..., accommodant en son milieu un espace pour la porte. Le diamètre intérieur de $\mathrm{X}$... doit être égal à la hauteur de la colonne sans le piédestal. Les colonnes qui courent autour de X... ont les mêmes proportions que celles du monoptère.

Il existe une quantité innombrable de $\mathrm{X}$..., et bien que leurs proportions soient identiques, ils sont néanmoins 
différents à cause de leur disposition: il en est ainsi du X... dédié à Castor dans le cirque Flaminien, et de celui de Vejovis, ou de Diane dans la forêt d'Aricie, lequel a des colonnes supplémentaires à droite et à gauche aux côtés du porche. Or, la manière dont est bâti le X... de Castor a été aussi pratiquée à Athènes pour Minerve, et dans l'Attique, pour Pallas: de proportions semblables, ils sont à l'intérieur deux fois plus longs que larges, et on a ajouté aux côtés ce que les autres (X...) ont sur la face avant.

Il y a aussi des X... qui ont été bâtis suivant l'ordre toscan, quoiqu'ils fussent d'ordre corinthien ou ioniques. Ainsi, les X... dont les murs s'avancent des deux côtés vers les pilastres pour en délimiter le porche possèdent deux colonnes à l'extrémité des murs, lesquels séparent le porche du X...: bel exemple de confusion de l'ordre toscan et de l'ordre corinthien.

D'autres X..., encore, élargis de l'intérieur, et sans que les proportions de l'ensemble ne changent, ont une configuration et un nom nouveau: ce sont les pseudopériptères. Les changements y ont été introduits pour la commodité des sacrifices, car il n'est pas concevable de sacrifier à tous les dieux des X... d'une même manière, à cause de la diversité des cérémonies, particulières à chacun d'eux.

J'ai décrit toutes sortes de X..., comme je les ai lues; j'ai distingué les ordres auxquels ils appartiennent et les proportions qui leur conviennent; j'ai aussi essayé d'expliquer en quoi leurs figures sont différentes les unes des autres.

Qui sont X...?

D'après M.P. Vitruve, De Architectura, chapitre VII, livre IV, corrigé et traduit par Claude Perrault, 1684. 